\title{
Haptic discrimination of bilateral symmetry in 2-dimensional and 3-dimensional unfamiliar displays
}

\author{
SOLEDAD BALLESTEROS \\ Universidad Nacional de Educación a Distancia, Madrid, Spain \\ DIONISIO MANGA \\ Universidad Computense, Madrid, Spain \\ and \\ JOSE MANUEL REALES \\ Universidad Nacional de Educación a Distancia, Madrid, Spain
}

\begin{abstract}
In five experiments, we tested the accuracy and sensitivity of the haptic system in detecting bilateral symmetry of raised-line shapes (Experiments 1 and 2) and unfamiliar 3-D objects (Experiments 3-5) under different time constraints and different modes of exploration. Touch was moderately accurate for detecting this property in raised displays. Experiment 1 showed that asymmetric judgments were systematically more accurate than were symmetric judgments with scanning by one finger. Experiment 2 confirmed the results of Experiment 1 but also showed that bimanual exploration facilitated processing of symmetric shapes without improving asymmetric detections. Bimanual exploration of 3 -D objects was very accurate and significantly facilitated processing of symmetric objects under different time constraints (Experiment 3). Unimanual exploration did not differ from bimanual exploration (Experiment 4), but restricting hand movements to one enclosure reduced performance significantly (Experiment 5). Spatial reference information, signal detection measures, and hand movements in processing bilateral symmetry by touch are discussed.
\end{abstract}

In the field of haptics, stimulus properties of shape other than stimulus extent, orientation, and curvature have received little attention (see Appelle, 1991, for a review). Most experimental studies on tactual perception have investigated cutaneous sensitivity (e.g., Bolanowski, Gescheider, Verrillo, \& Checkosky, 1988), and a smaller number have focused on the properties of haptic performance by active exploration.

This research was supported by DGICYT Research Grant PB900003 and by a UNED Grant. J.M.R. was supported by a Universidad Nacional de Educación a Distancia graduate fellowship. The Spanish Organization for the Blind (ONCE) prepared the haptic 2-D materials used in these experiments. S.B. presented portions of this research at the 33rd annual meeting of the Psychonomic Society, St. Louis, November 1992, and at the International Conference on Object Representation in Visual and Haptic Systems, Madrid, May 1993. We are very grateful to the participants in the conference for their very helpful comments. The authors wish to thank especially R. L. Klatzky for comments on an earlier version of this paper. S.B. wants to thank S. Millar for very helpful discussions on spatial coding and haptic perception during the conference, and particularly during our collaborative research project. We are also very much in debt to L. Marks for his very helpful comments on an earlier version of this manuscript. Finally, the authors are very grateful to $\mathrm{M}$. L. Braunstein and two anonymous reviewers whose comments helped to improve the paper greatly. Correspondence should be addressed to S. Ballesteros, Departamento de Psicología Básica II, Universidad Nacional de Educación a Distancia, P.O. Box 50879, Madrid, Spain (e-mail: sballast@cu.uned.es).
The haptic system is considered a complex perceptual system that encodes inputs from cutaneous and kinesthetic receptors (Loomis \& Lederman, 1986). As far as we know, no one has focused yet on haptic processing of bilateral symmetry of novel displays other than plane polygons or raised dots. The study of symmetry in haptic shapes has been neglected. Even Katz (1925/1989), a pioneer in the study of touch, did not consider haptic perception of this invariant property of form in his monograph. Révész (1950) suggested that symmetry judgments are recognized, but are not based on immediate sensory apprehension. According to Révész, haptic perception of symmetry occurs through movements. After presenting several observations of closed-outline, raised-planar shapes, he found that haptic perception of symmetry for solid and other higher properties of form is very poor. Recent research has shown that the haptic system is not as good at processing raised-pattern information as it is at perceiving (Klatzky, Lederman, \& Metzger, 1985) and remembering 3-D familiar and unfamiliar objects.

Millar (1978) conducted a pioneer investigation on haptic perception of symmetry using raised-dot symmetric and asymmetric patterns of five and nine dots. She found that the detection of differences in symmetry was poorer than the detection of differences in dot numerosity ("texture," as she called it). Millar suggested that subjects relied on texture differences because stimuli provided insufficient 
reference information for spatial coding of shape. According to Millar, when subjects explore unfamiliar raised shapes for which they have no spatial referents, differences in texture instead of differences in the spatial organization of shape are coded. Furthermore, Klatzky, Lederman, and Reed (1987) showed that in haptic exploration, properties such as texture and hardness are more salient than is information about form and size.

Locher and Simmons (1978) investigated the detection of the symmetric or asymmetric characteristics of individually presented planar polygons that also varied in structural complexity as measured by the number of sides (varying from 12 to 30 ). Using only eight different very large planar polygons, they found that the detection of asymmetry was faster than the detection of symmetry at all complexity levels. The number of incorrect identifications was also greater for symmetric than for asymmetric shapes ( $11.8 \%$ vs. $2.6 \%$, respectively). In another study, Simmons and Locher (1979) showed that scanning time and number of errors decreased with perceptual experience. Furthermore, simultaneous scanning strategies produced greater sensitivity as measured by the index $d^{\prime}$ (Signal Detection Theory; SDT) with symmetric polygons. Trace scanning strategy resulted in a greater sensitivity with asymmetric polygons. However, although a simultaneous scanning resulted in greater sensitivity for symmetric polygons, it did not decrease exploration time. Symmetric shapes required twice as much decision time as did asymmetrical shapes.

The present experiments focus on the capacity of the haptic system to detect bilateral symmetry in unfamiliar raised-line shapes as well as in 3-D novel objects. Comparison of performance with 2-D and 3-D displays is important because, as Lederman and Klatzky (1987) showed, in contrast to the poor performance with raised displays, familiar 3-D objects are recognized very efficiently by touch. The difference in performance has been explained in terms of movement information, which is greater for objects than for raised displays. More recently, Klatzky, Loomis, Lederman, Wake, and Fujita (1993) investigated different explanations of the real-object superiority effect in an identification task using real objects and their raised outline drawings. They found that the contribution of 3-D structure and the integration of cues across the fingers were major factors in performance.

In the present experiments, we explored the influence of constraining exploration time and the use of a bodycentered reference frame on the discrimination of symmetry in 3-D objects and 2-D raised line displays. We are aware that it is not possible to compare 3-D and 2-D stimuli directly because the stimuli differed primarily in size and complexity. Our intention was not to provide a comparison between 2-D versus 3-D performance. For that reason, in designing the stimuli we considered which was the most suitable size of a raised shape to be explored by the fingertip and the more convenient size for objects to be explored by two hands.

The purpose of the series of experiments reported in this paper was twofold: (1) to assess the sensitivity of the haptic system in the detection of bilateral symmetry in 2-D raised displays as a function of scanning time and mode of exploration (unimanual vs. bimanual); and (2) to investigate the capacity of the haptic system to detect bilateral symmetry in 3-D objects by assessing its accuracy and sensitivity. As far as we know, no one has yet focused on haptic processing of symmetry in 3-D objects. As in raised shapes, we considered the influence of restricting the exploration time and the possible advantage of using two hands versus one hand in the detection of bilateral symmetry. Finally, we analyzed the type of hand movements performed during object haptic exploration.

The detection of the symmetry/asymmetry in raisedline shapes was studied in Experiments 1 and 2. In Experiments 3,4 , and 5 , we explored haptic detection of bilateral symmetry in 3-D objects.

\section{EXPERIMENT 1}

In Experiment 1, we investigated the perceptual sensitivity of the haptic system in detecting bilateral symmetry in raised-line shapes. The main aim was to study the effect of exploration time on haptic performance. Three exploration times were used: free exploration, $5 \mathrm{sec}$, and $2 \mathrm{sec}$.

\section{Method}

Subjects. Subjects were 60 undergraduate students at the Universidad Nacional de Educación a Distancia (UNED) and at the Computense University of Madrid. All subjects were right-handed according to the Edinburgh Inventory (Oldfield, 1971).

Stimuli. The shapes were carefully designed to be suitable for discrimination by touch. A $3 \times 3$ dot matrix was found to conform best to the requirement that raised shapes should be simple and legible. The stimuli consisted of 48 patterns constructed by connecting five or six dots in the matrix. Half of the stimuli were symmetric about the vertical axis of symmetry only. The other half were asymmetric. These stimuli were a subset of the total 2-D set used by Ballesteros and Cooper (1992) in a visual study. Examples of the stimuli used in Experiments 1 and 2 are shown in Figure 1.

All raised-line shapes were then prepared professionally for haptic presentation by the Spanish Blind Organization (ONCE). The shapes, prepared in thermoform, appeared in the center of a $20 \mathrm{~cm}$ by $14 \mathrm{~cm}$ card and measured approximately $2 \mathrm{~cm} \times 2 \mathrm{~cm}$. The raised lines were $2.5 \mathrm{~mm}$ wide $\times 1.2 \mathrm{~mm}$ high. The shapes were displayed

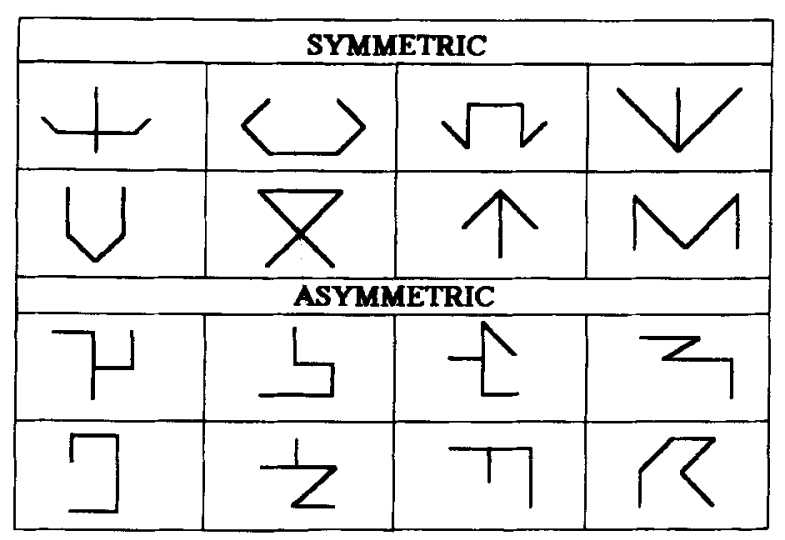

Figure 1. Examples of symmetric and asymmetric raised-line shapes used in Experiments 1 and 2. 
individually in random order for each subject and were presented lying in a horizontal plane in front of the observer midaxis.

Design and Procedure. The experimental design was a 3 (exploration times: unlimited, $5 \mathrm{sec}$, and $2 \mathrm{sec}$ ) $\times 2$ (types of shape: symmetric and asymmetric) factorial with repeated measures on the last factor. The task was to judge whether the shapes were symmetric or asymmetric.

Subjects were tested singly in the laboratory. They were blindfolded and seated directly in front of the table that supported the stimulus card. The midaxis of the stimulus card was aligned to the body midaxis of the subject in the midtransverse plane. The difference between symmetric and asymmetric shapes was explained, and subjects were given examples of symmetric and asymmetric shapes to feel. All subjects explored each pattern with their right index fingertip. Twenty observers were allocated randomly to the three exploration time conditions: unlimited, $5 \mathrm{sec}$, and $2 \mathrm{sec}$. Subjects were asked to respond as quickly and accurately as possible.

\section{Results and Discussion}

Table 1 shows mean accuracy of judgments. The data were analyzed first in terms of accuracy and then in terms of sensitivity and response bias (SDT).

Accuracy analysis. The overall accuracy was $70 \%$. Asymmetric shapes were judged more accurately (81\%) than were symmetric shapes $(60 \%)$. The haptic system appeared to be as accurate after 2-sec exploration as at longer exploration times. ${ }^{1}$ The analysis of variance (ANOVA) conducted on the accuracy scores showed that the main effect of shape (symmetric vs. asymmetric) was highly significant $\left[F(1,57)=31.13, M S_{\mathrm{e}}=407.49, p<.001\right]$. There was no significant effect of exploration time or an interaction between exploration time and type of shape (both $F \mathbf{s}<1$ ).

Sensitivity and response bias analysis. Sensitivity measures were computed from the individual symmetric and asymmetric judgments. The sensitivity and bias indexes, $d^{\prime}$ and $c$, were calculated from $z$-score transformations of the mean proportions of hits ("symmetric" responses to symmetric targets) and false alarms ("symmetric" responses to asymmetric targets). These parameters were calculated for each subject using the SDT_SP computer program (Reales \& Ballesteros, 1994, 1995). Individual estimates were then averaged. Results displayed in Table 1 show observer sensitivity and bias under the three exploration time conditions.

As a measure of response bias, in this and subsequent experiments, the basic bias measure for SDT, called $c$

Table 1

Symmetry/Asymmetry Performance, Accuracy, and Signal Detection Analysis: Experiment 1

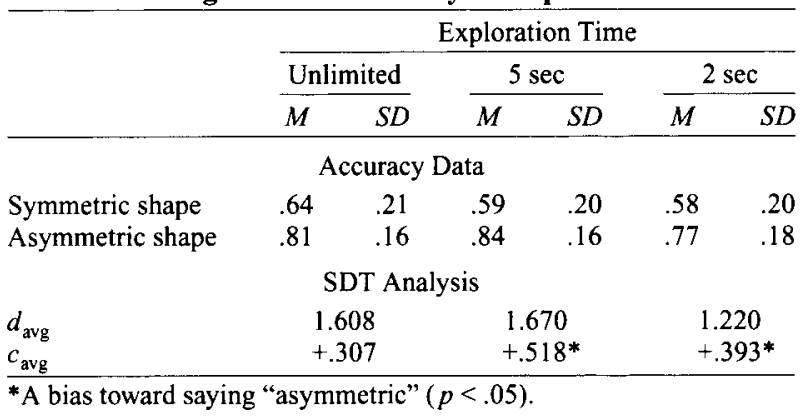

(for "criterion"), was calculated. We chose this measure because it has the simplest statistical properties of the alternative measures derived from SDT (see Macmillan \& Creelman, 1991). The bias parameters $c$ were $.307, .518$, and .393 for unlimited, 5-sec, and 2-sec exploration, respectively. The last two values were significantly different from 0 . Positive values of $c$ indicate a bias to respond "asymmetric." The results indicate that the exploration time did not significantly affect performance, consistent with the lack of main effects in the accuracy ANOVA. However, the SDT analysis showed that for unlimited exploration time, there was no significant response bias. By contrast, there was a response bias in the 5-sec and 2-sec exploration conditions, although the ANOVA did not show it (Table 1).

Loomis (1990) obtained a similar level of accuracy $(70 \%)$ in a character recognition task using Braille letters and line patterns based on Braille dots matrix with $2 \mathrm{sec}$ of exploration. We obtained the same level of accuracy for our symmetry/asymmetry detection task and found that observers were about $20 \%$ more accurate at detecting asymmetric shapes than symmetric shapes.

To summarize, the SDT analysis showed that the sensitivity of the haptic system in the symmetry detection task was acceptable ( $d_{\text {avg }}^{\prime}$ around 1.5) when subjects explored the shapes using the index fingertip of their preferred hand. The advantage for asymmetric detection cannot be attributed to response bias. Free exploration showed no significant response bias, but performance was nevertheless significantly more accurate for asymmetric detections $(82 \%)$ than for symmetric detections $(64 \%)$.

\section{EXPERIMENT 2}

In stationary blindfolded conditions, there is little information about external frames for coding small stimuli. According to Millar (1994), in these conditions information is restricted to "personal" space. The body midline or body axis provides invariant proprioceptive information to which haptic stimuli are referred.

Millar $(1979,1981,1985)$ conducted several studies on the effects of self-referent and movement cues in coding spatial locations. She suggested that both types of cues are important and that the information gathered by these two types of cues differs. In this experiment, we explored the effect of bilateral versus unilateral perceptual processing on the haptic detection of symmetry. In the reference hypothesis, it is assumed that the effect of symmetry depends on the available reference information. The lesser salience of symmetry in touch is explained by the paucity of reference cues (Millar, 1994). In blind conditions, spatial coding depends on reference frames that are centered on body coordinates. Thus we asked observers to explore shapes with two fingers, using the right and left forefingers simultaneously. A further two groups explored the shapes only with the index fingerpad of the right or of the left hand. We expected that bimanual exploration would provide the perceiver with important information derived from two parallel movements executed 
in relation to a body-centered reference frame. This new information could help in coding the spatial arrangement of tangible shapes in relation to its vertical/central axis of symmetry. In the reference hypothesis, it is predicted that bimanual exploration will produce better performance with symmetric shapes than does unimanual exploration.

\section{Method}

Subjects. Forty new observers participated in this experiment. They were students from the same pool as those in the first experiment. They participated for course credit. Twenty subjects were randomly allocated to the bimanual exploration, and the other 20 were tested with the left index finger. The data from the 20 observers who participated in Experiment 1 in unimanual right exploration of shape were used in the analysis.

Material and Stimuli. The stimuli were the same as those in Experiment 1. To run the experiment and record the data of interest, a piezoelectric board was interfaced with an IBM System $/ 2$ computer. The piezosensor was located in the center beneath the board external surface. The computer program provided a random order of raised shapes for each subject and recorded the data.

Design and Procedure. The experimental design was a 3 (exploration: bimanual/left forefinger/right forefinger) $\times 2$ (type of shape: symmetric and asymmetric) factorial with repeated measures on the last factor. Exploration time was free in all the experimental conditions. On each trial, the experimenter placed the randomly selected shape in the center of the board. A tone from the computer alerted the subject that the stimulus was in place. The experimenter recorded the subject's response by pressing the appropriate key on the computer keyboard. Between trials, the subject rested the two index fingers in two holders located on the base of the board. After hearing the starting signal, the subject moved the fingers from the starting position to the raised shape.

The dependent variable was accuracy. Instructions and the rest of the procedure were the same as those in Experiment 1.

\section{Results and Discussion}

As in the previous experiment, we report first the accuracy results and then the sensitivity and response bias analysis. The mean accuracy data are shown in Table 2 .

Accuracy analysis. The ANOVA on correct responses showed a significant main effect of mode of exploration $\left[F(2,57)=3.27, M S_{\mathrm{e}}=183.24, p<.05\right]$. Post hoc comparisons showed that bimanual accuracy $(81 \%)$ was significantly higher than left finger exploration $(73 \%, p<$ $.05)$. The comparison between the right $(75 \%)$ and left index finger accuracy, however, did not reach statistical significance. As in Experiment 1, type of shape was highly

Table 2

Symmetry/Asymmetry Performance, Accuracy, and Signal Detection Analysis: Experiment 2

\begin{tabular}{|c|c|c|c|c|c|c|}
\hline & \multicolumn{6}{|c|}{ Mode of Exploration } \\
\hline & \multicolumn{2}{|c|}{ Right $F$} & \multicolumn{2}{|c|}{ Left $F$} & \multicolumn{2}{|c|}{ Two $\mathrm{F}$} \\
\hline & $M$ & $S D$ & $M$ & $S D$ & $M$ & $S D$ \\
\hline \multicolumn{7}{|c|}{ Accuracy Data } \\
\hline Symmetric shape & .63 & .16 & .60 & .19 & .76 & .16 \\
\hline Asymmetric shape & .87 & .11 & .86 & .12 & .86 & .09 \\
\hline \multicolumn{7}{|c|}{ SDT Analysis } \\
\hline$d_{\text {avg }}$ & \multirow{2}{*}{\multicolumn{2}{|c|}{$\begin{array}{l}1.852 \\
+.559^{*}\end{array}$}} & \multirow{2}{*}{\multicolumn{2}{|c|}{$\begin{array}{l}1.765 \\
+.578^{*}\end{array}$}} & \multirow{2}{*}{\multicolumn{2}{|c|}{$\begin{array}{l}2.082 \\
+.170\end{array}$}} \\
\hline$c_{\text {avg }}^{\text {avg }}$ & & & & & & \\
\hline
\end{tabular}

*A bias toward saying "asymmetric" $(p<.05) ; \mathrm{F}$, forefinger. significant $\left[F(1,57)=54.54, M S_{\mathrm{e}}=222.94, p<.001\right]$. The mean accuracy for the symmetric shapes was $66 \%$ versus $86 \%$ for the asymmetric shapes. The interaction mode of exploration $\times$ type of shape $[F(2,57)=3.80$, $\left.M S_{\mathrm{e}}=222.94, p<.05\right]$ was also significant. This interaction showed that the bimanual advantage was due to the better performance with the symmetric shapes. Nevertheless, even bimanual accuracy was higher for asymmetric than for symmetric shapes (Figure 2).

These results showed if anything that left hand did not improve performance in this spatial task. One interesting result that confirmed our prediction was that the improved performance for bimanual compared to unimanual exploration occurred only with symmetric shapes. That is to say, symmetric shapes explored by the two forefingers were detected more accurately than when the same shapes were explored with the left forefinger. By contrast, performance with asymmetric shapes did not change with the mode of exploration. These results are in accordance with the body axis reference frame hypothesis. Parallel information coming from the two fingers favors shape space coding in relation to the body midline, improving symmetric detection. Symmetry in touch seems to depend on the amount of the reference information that is available for spatial coding. Facilitating effects of symmetry were found in a condition designed to enhance reference bodycentered information.

Similar results were found in a series of studies in which stimulus orientation of symmetric and asymmetric open and closed shapes (vertical, horizontal, and oblique) and mode of exploration (one handed vs. two handed) were systematically manipulated. The subjects' task was to judge stimulus closure rather than to detect symmetry (Ballesteros, Millar, \& Reales, 1996; Millar, Ballesteros, \& Reales, 1994).

Sensitivity and response bias analysis. The sensitivity and response bias measures are shown in Table 2. Note that there was not a response bias in the bimanual mode of exploration, whereas a significant bias was shown in the two unimanual exploration conditions. Table 2 shows the hit and false-alarm rates averaged across subjects. Sensitivity $\left(d_{\text {avg }}^{\prime}\right)$ and bias $(c)$ for each subject were calculated and then averaged. Haptic sensitivity, measured as $d_{\text {avg }}^{\prime}$, was 2.08 , and bias, measured as $c_{\text {avg }}$, was .17. Sensitivity improved and bias decreased in the two-finger condition compared with one-finger conditions. Note, however, that asymmetric performance was more accurate than symmetric performance over all experimental conditions. These results, overall, confirmed the results of Experiment 1 . The haptic system processes asymmetric raised shapes more easily than symmetric ones.

Our results agree with those of Lappin and Foulke (1973), who found that performance was better when the displays were scanned by two fingers on different hands. These researchers interpreted this result as indicating the occurrence of parallel processing of the perceptual inputs from the two fingers, one from each hand. Craig (1985) also found that performance improved when vibrotactile patterns were presented to the two fingers on opposite 


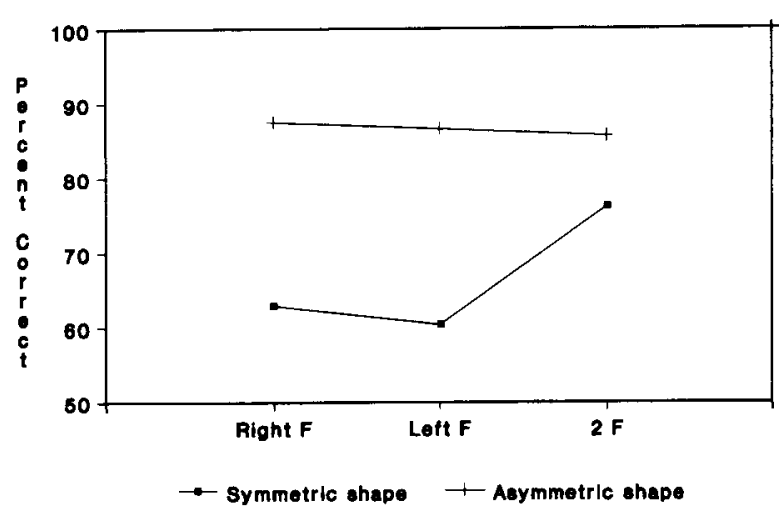

Figure 2. Mean accuracy scores for symmetric and asymmetric shapes under three modes of exploration: right, left, and two forefingers (Experiment 2).

hands relative to two fingers in the same hand. However, neither study investigated haptic perception of symmetry.

To summarize, bimanual exploration in relation to a body midline benefited the detection of symmetric shapes without changing the detection of asymmetric shapes. This result is in accordance with the reference frame hypothesis.

\section{EXPERIMENTS 3-5}

The aim of Experiments 3-5 was to examine the perception of symmetry with 3-D objects. According to Lederman and Klatzky (1987), the discrepancy between the poor haptic performance obtained with 2-D depictions of raised shapes compared with the outstanding performance with common multidimensional 3-D objects can be reconciled by taking into account the type of movements performed during haptic exploration of 2-D and 3-D stimuli. Similarly, Heller (1992) has argued that it is inappropriate and "unfair" to compare optimal conditions on vision with poor conditions on touch. Threedimensional objects are usually manipulated by the human hand in our daily experience with the world. Nevertheless, haptic perception of 3-D objects has rarely been examined in laboratory studies (see Appelle, 1991); for some recent studies on haptic discrimination of surfaces of solid objects, see Kappers, Koenderink, and Lichtenegger (1994) and Kappers, Koenderink, and te Pas (1994). Davidson, Abbott, and Gershenfeld (1974) compared intra- and cross-modal matching of Gibson form solids and showed large effects of exploration time (4-16 sec) on accuracy in haptic/visual matching tasks. Furthermore, Garvin and Bernstein (1984) found that subjects categorize solid forms in terms of metric properties such as symmetry and complexity.

In order to probe the detection of symmetry in the haptic system under conditions of richer information than is provided by raised-line displays, we used 3-D manipulable wooden objects in Experiments 3, 4, and 5. The task, as in the previous two experiments, was direct detection of symmetry. We expected that the haptic system would be very efficient in detecting this spatial-structural property of shape in 3-D objects. The reason for this is that rich and free hand movements plus body-centered reference frames will provide touch with more appropriate spatial information. Furthermore, exploring this type of stimuli with the whole hand, instead of just the fingertip, would provide simultaneous parallel inputs to the system. Bimanual exploration should therefore favor parallel perceptual processing. Three-dimensional objects allow the haptic system to use its motor component fully as well as its sensory component (Lederman \& Klatzky, 1987). Instead of simply scanning or tracing successively the display with just the fingertip, as in the raised shapes, observers would be able to explore the objects with all the fingers of (one or) both hands simultaneously. In other words, haptic observers might feel our solid 3-D objects and perceive their configuration through active touch using a more holistic strategy. Furthermore, observers could use their body axis as a spatial reference frame to locate features of the right and the left side of the objects. We expected this strategy to benefit the detection of symmetric objects.

\section{Experiment 3}

The aim of Experiment 3 was to assess the accuracy and sensitivity of active touch as well as the hand movements performed during the symmetry task over a range of exploration time conditions. According to Lederman and Klatzky (1987), the exploratory procedures related to detecting the shape of 3-D objects are "enclosure" and "contour following." By restricting the exploration time, we try to test the hypothesis that the number of the enclosure exploratory movements executed to detect the symmetry/asymmetry of haptic objects would increase, and the number of contour following movements would decrease, when exploration time is limited. It was anticipated that a general purpose movement, such as enclosure, consisting of molding the hands around the object (see Klatzky \& Lederman, 1992; Lederman \& Klatzky, 1987,1990 ), would provide the system with simultaneous information from the apex, edges, and surfaces. This type of movement would suffice to apprehend bilateral symmetry as an invariant relation between both sides of the objects and the perceiver's body axis.

\section{Method}

Subjects. Eighty sighted undergraduate students at the Computense and the UNED universities participated for course credit; none had participated in the previous studies. All reported to be right-handed. Subjects were allocated randomly to the experimental conditions.

Stimuli and Apparatus. The stimuli used in these experiments were 3-D unfamiliar objects made of wood. Five of them were haptic replicas of the visual objects used by Schacter, Cooper, and associates (see Cooper, Schacter, Ballesteros, \& Moore, 1992; Schacter, Cooper, \& Delaney, 1990) in studies of visual priming. The other 35 objects were specially designed for this study. The size of the objects was selected on the basis of its facility of exploration by 
an adult, standard-size hand. Examples of symmetric and asymmetric objects are shown in Figures 3 and 4, respectively.

The stimuli consisted of 40 unfamiliar wooden objects, 20 bilaterally symmetric and 20 asymmetric. They were made from a cubic piece of wood approximately $7 \mathrm{~cm}$ long $\times 7 \mathrm{~cm}$ wide $\times 6 \mathrm{~cm}$ high. The symmetric objects were bilaterally symmetrical. The equipment was the same as that in Experiment 2.

In experimental conditions with restricted exploration time, a second auditory signal informed subjects that they should stop touching the object. The hand movements were videotaped by a video camera attached to the wall, located in front of the perceiver. A monitor completed the hand-recording equipment. Hand movements were analyzed by frame-by-frame analysis.

Design and Procedure. The design was a 4 (exploration times: unlimited, $5 \mathrm{sec}, 3 \mathrm{sec}$, and $1.5 \mathrm{sec}$ ) $\times 2$ (type of object: symmetric and asymmetric) mixed factorial. The first factor was manipulated between subjects. The second factor was within subjects. Observers were seated in front of the display board and were blindfolded. They

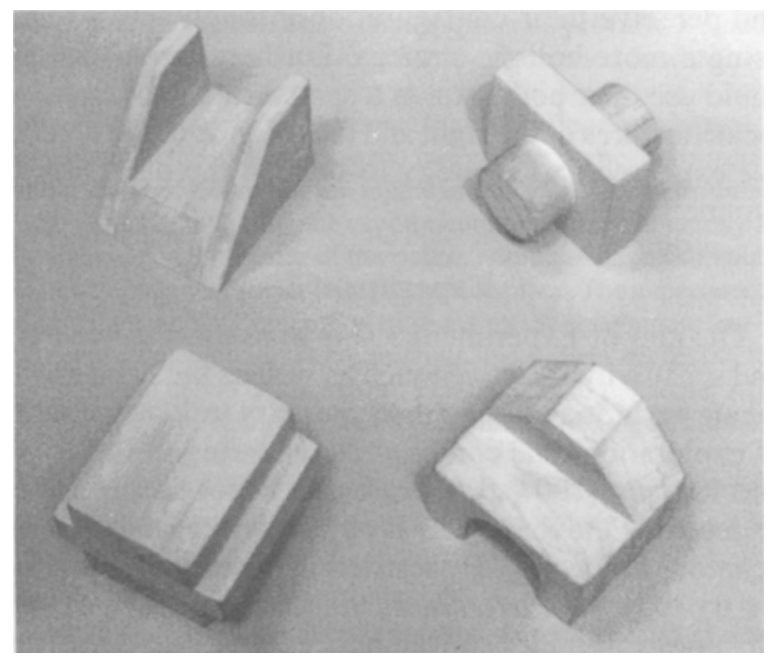

Figure 3. Examples of the unfamiliar symmetric wooden objects used in Experiments 3-5.

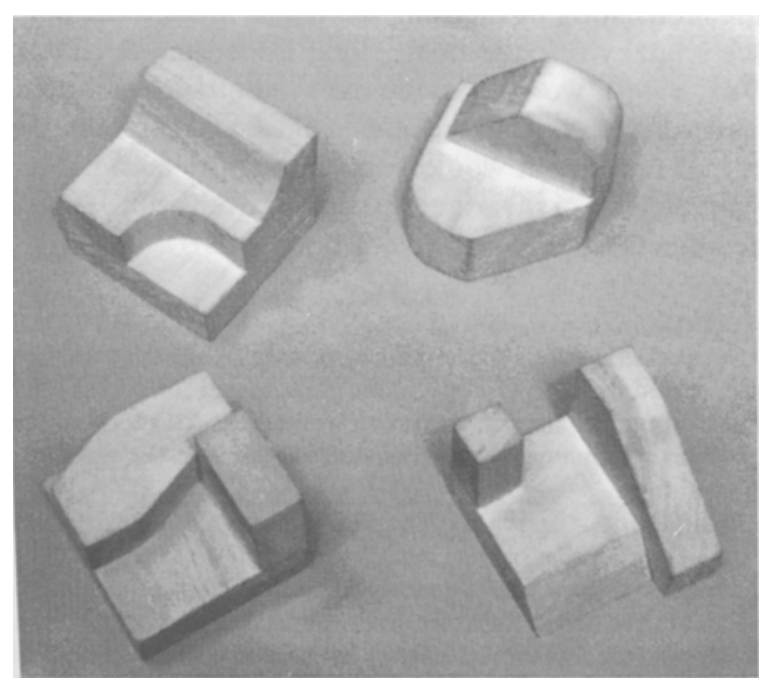

Figure 4. Examples of the unfamiliar asymmetric wooden objects used in Experiments 3-5. were tested individually in a quiet room. The objects were always kept out of sight. Observers were told that they were participating in an experiment on object perception by touch. The objects were displayed one at a time, randomly determined for each subject by the computer. Each object was presented in the piezoelectric board located on the table at which the subject was seated. The object was placed on the center of the board aligned to the observer's body midline. The order of presentation was determined randomly for any subject by computer program. The randomly selected object was placed on the center of the board so that its axis of symmetry was vertical and parallel with respect to the frontal plane of the subject's body. Instructions were the same as those in Experiment 1. Observers were asked to explore the objects with both hands simultaneously. Time limits were not imposed on participants in the free exploration condition. In the other three conditions, exploration time was constrained to 5, 3, and $1.5 \mathrm{sec}$, respectively. Twenty subjects were tested in each condition. Observers were asked to speak clearly into the attached microphone as quickly and accurately as possible whether the object was bilaterally "symmetric" or "asymmetric." They were asked not to pick the object up or to rotate it. Four practice trials were given, followed by 40 experimental trials. Response times were recorded from the time the hands first contacted the object to the verbal response.

\section{Results and Discussion}

Mean accuracy judgments are shown in Table 3 . These results are described first in terms of accuracy and then in terms of sensitivity and bias.

Accuracy and response time analysis. The overall accuracy was $85 \%$. Mean percentage of accuracy results were, respectively, $90 \%, 87 \%, 86 \%$, and $76 \%$ for the unlimited, 5-, 3-, and 1.5-sec exploration time conditions (Figure 5). Observers were more accurate in detecting symmetric objects than asymmetric objects $(p<.001)$. An important point should be made about this result. As predicted, the haptic perceptual system was very efficient. The ANOVA on accuracy judgment scores showed a significant main effect of exploration time $[F(3,76)=7.59$, $\left.M S_{\mathrm{e}}=191.60, p<.001\right]$. Newman-Keuls analysis showed that differences between the three time conditions (unlimited, $5 \mathrm{sec}$, and $3 \mathrm{sec}$ ) differed significantly from the 1.5 -sec presentation time $(p<.01)$. The other presentation time conditions did not differ significantly. The result showed that haptic detection of symmetry in 3-D objects was worse at the shortest exploration time but did not differ at presentation times longer than $3 \mathrm{sec}$. The main effect of symmetry was also significant $[F(1,76)=$ $\left.20.136, M S_{\mathrm{e}}=176.93, p<.001\right]$. The interaction between these two factors was not significant $(F<1)$.

The mean response time was $9.1 \mathrm{sec}$, and the difference between symmetrical and asymmetrical objects ( 9.4 vs. $8.8 \mathrm{sec}$, respectively) was not statistically significant $(F<1)$.

Sensitivity and bias analysis. Table 3 shows the signal detection results of sensitivity $\left(d_{\text {avg }}^{\prime}\right)$ and bias. The sensitivity indexes for all exploration conditions were highfrom 3.279 (free exploration) to 1.810 (1.5-sec exploration). Contrast showed differences between all pairs of means and $1.5 \mathrm{sec}(p<.05)$. That means that sensitivity decreased considerably when exploration time was restricted to $1.5 \mathrm{sec}$.

The values of $c$ as a measure of bias showed that the only condition in which this parameter was significantly 
Table 3

Symmetry/Asymmetry Performance, Accuracy, and Signal Detection Analysis: Experiment 3

\begin{tabular}{|c|c|c|c|c|c|c|c|c|}
\hline & \multicolumn{8}{|c|}{ Exploration Time } \\
\hline & \multicolumn{2}{|c|}{ Unlimited } & \multicolumn{2}{|c|}{$5 \mathrm{sec}$} & \multicolumn{2}{|c|}{$3 \mathrm{sec}$} & \multicolumn{2}{|c|}{$1.5 \mathrm{sec}$} \\
\hline & $M$ & $S D$ & $M$ & $S D$ & $M$ & $S D$ & $M$ & $S D$ \\
\hline \multicolumn{9}{|c|}{ Accuracy Data } \\
\hline Symmetric object & .93 & .08 & .90 & .11 & .90 & .15 & .83 & .14 \\
\hline Asymmetric object & .86 & .12 & .83 & .10 & .82 & .19 & .68 & .16 \\
\hline \multicolumn{9}{|c|}{ SDT Analysis } \\
\hline$d_{\mathrm{avg}}$ & \multirow{2}{*}{\multicolumn{2}{|c|}{$\begin{array}{c}3.279 \\
-.221\end{array}$}} & \multicolumn{2}{|c|}{2.760} & \multicolumn{2}{|c|}{2.974} & \multicolumn{2}{|c|}{1.810} \\
\hline$c_{\text {avg }}^{\text {avg }}$ & & & \multicolumn{2}{|c|}{-.295} & \multicolumn{2}{|c|}{-.285} & \multicolumn{2}{|c|}{$-.335^{*}$} \\
\hline
\end{tabular}

different from zero was the 1.5 -sec exploration ( $c=$ $-.335, S D=.196$ ). Bias did not differ from zero in the other three conditions.

Three important points should be noted. First, accuracy with 3-D objects was very high-in fact, higher than with raised patterns under different time constraints. Second, as expected, accuracy for symmetric objects was higher than that for asymmetric objects. Third, performance was moderate even with an exploration time as short as $1.5 \mathrm{sec}$, even though it was poorer than in the other three exploration time conditions. The results of the detection theory analysis are consistent with the ANOVA on accuracy discussed above.

Haptic detection of symmetry with 3-D objects was thus very accurate and sensitivity measured by the SDT parameter $d^{\prime}$ was also high except for the 1.5 -sec exploration condition. It is important to mention that only with the shortest exploration was a bias to respond "symmetric."

One finding was that the advantage for asymmetric compared with that for symmetric shapes found in Experiments 1 and 2 was reversed in judging 3-D objects. Performance with 3-D objects was more accurate than that for raised shapes. With 3-D objects, observers seem to approach the detection of symmetry by using the two hands together. By contrast, the exploration, even bimanually, with the raised-line shapes (Experiment 2 ) required successive movement. The more global exploration that is possible with 3-D objects allows more parallel extraction of information than is possible by sequential exploration. In the following section we report the hand movement analysis.

\section{Hand Movement Analysis}

Many researchers on touch have noticed the importance of movements performed during haptic exploration (e.g., Davidson, 1972; Gibson, 1962; Katz, 1925/1989; Locher \& Simmons, 1978; Zinchenko \& Lomov, 1960). Most relevant to the present study is the approach to hand movement analysis provided by Lederman and Klatzky (Klatzky \& Lederman, 1992; Lederman \& Klatzky, 1987, 1990). According to these investigators, hand movements can be considered as the "windows" through which we can learn about the underlying representation of objects and the psychological processes that manipulate these representations. Lederman and Klatzky have shown that observers actively explore objects with their hands, executing a number of stereotyped hand movements called "exploratory procedures" in searching for salient attributes or trying to identify common objects apprehended haptically. Each of these hand movements is associated with a particular object dimension. For example, Lederman and Klatzky clearly differentiated three patterns of hand movements directly associated with the apprehension of structural properties of objects. These classes of movements included enclosure (body), a movement consisting of adapting the palm of the hand and fingers to object contours, and associated with the dimensions of volume and gross shape; and contour following, consisting of the dynamic exploration along the edges and surfacesthis movement was associated with gross shape and with the apprehension of precise shape. Lederman and Klatzky (1990) further differentiated a type of enclosure that they called "enclosure (part)." This movement consisted of molding the fingers to a secondary part of the object instead to the whole object; it was also associated with shape. Other classes of movements, such as lateral motion, pressure, static contact, and unsupported holding, were associated with substance properties of objects, such as texture, hardness, and temperature.

The main goal of the present analysis was to try to understand how object manipulation leads to the detection of symmetry as a property of object structure. We envision this approach as a way to try to explain the difference in haptic performance observed in this and other studies that have used raised 2-D displays and 3-D objects. Thirty-three subjects were selected randomly and their exploration data were analyzed.

The four main predictions were as follows:

1. As symmetry is a structural property of shape, according to Lederman and Klatzky $(1987,1990)$, we expected that the class of movements mostly executed by our haptic explorers would be the three reported by these investigators as related to the apprehension of shape information: global enclosure, contour following, and enclosure (part).

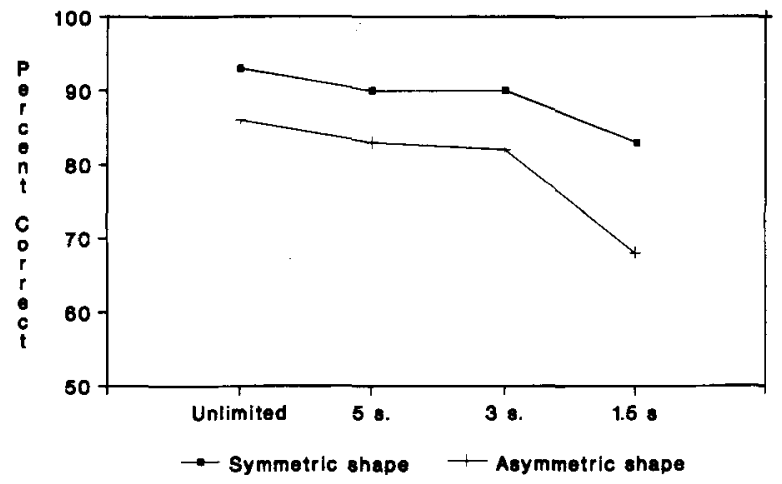

Figure 5. Mean accuracy scores for symmetric and asymmetric objects under four exploration times (Experiment 3 ). 
2. Simultaneous exploration movements should increase in number as the time allowed to explore the object decreases.

3. Global enclosure (the most global exploratory procedure) should be the most frequently executed movement in detecting symmetry as exploration time is constrained. The enclosure (part) movement was expected only in exploring those objects with salient parts. This movement is not only a quick and general purpose movement, but it also allows parallel extraction of structural information about object shape.

4. The time spent on the successive exploration of object contours should diminish as the time becomes shorter, still maintaining adequate levels of performance.

The videotapes corresponding to 33 observers randomly selected from each exploration time condition were evaluated very carefully by a scorer who was naive with respect to the purpose of the experiments. We provided her with a written description of the exploratory procedures proposed by Lederman and Klatzky $(1987,1990)$, followed by a good amount of practice. For any trial, she recorded (1) the class of any exploratory movement performed, (2) the duration of each of the movements, and (3) its position in the sequence of total movements. All trials were assessed in terms of hand movements; however, only data corresponding to correct trials were considered in the analysis. According to Lederman and Klatzky, the basic unit of observation is the exploratory procedure that is a stereotyped pattern of movements exhibiting certain invariant or highly typical characteristics.

Accuracy analysis. We first checked the accuracy for each exploration time condition. As noted, when we reported the performance of all the participants, accuracy was high and decreased as exploration time declined: $94 \%, 85 \%, 84 \%$, and $77 \%$ for unlimited time $-3,5$, and $1.5 \mathrm{sec}$, respectively. A total of 2,386 movements corresponding to the correct trials across exploration time conditions were considered in the analysis.

As expected from the work of Lederman and Klatzky $(1987,1990)$, the three main types of movements observed were global enclosure, enclosure (part), and contour following. The number of other movements was insignificant (e.g., static contact and lateral motion, were very infrequent $-0.4 \%$ of total movements; $6.7 \%$ were double movements) and thus not further analyzed. Table 4 shows the proportion of type of movement as a function of exploration time. Note that the proportion of global enclosures performed as the first movement in the sequence increased with the reduction of exploration time. In the 1.5-sec condition, the proportion of movements in this category was more than half of the movements performed (.52). At the same time, the proportion of enclosure (part) and contour following movements decreased (.12 and .36, respectively). It seems that as the time allowed to explore the object became shorter, observers tried to execute the most global type of movement. According to Lederman and Klatzky, global enclosure is broadly sufficient for at least coarse discrimination of
Table 4

Proportion of Each Class of Movements Performed During Haptic Exploration of Symmetry as a Function of Exploration Time

\begin{tabular}{lccc}
\hline \multirow{2}{*}{$\begin{array}{c}\text { Type of } \\
\text { Movement }\end{array}$} & \multicolumn{3}{c}{ Exploration time } \\
\cline { 2 - 4 } & Unlimited time & $3-5 \mathrm{sec}$ & $\mathbf{1 . 5 \mathrm { sec }}$ \\
\hline Enclosure (global) & .37 & .38 & .52 \\
Contour following & .42 & .42 & .36 \\
Enclosure (part) & .21 & .20 & .12 \\
\hline
\end{tabular}

Note-Data from 3- and 5-sec exploration time conditions were collapsed.

shape properties. Furthermore, this movement was faster to execute than contour following.

Analysis of exploration times. The mean duration in seconds corresponding to the first time that a given type of movement appeared (global enclosure, enclosure [part], and contour following) in each trial was averaged across trials and observers for every exploration time condition independently.

For the purpose of the hand movement analysis (see Tables 4 and 5 and Figures 6 and 7), 3- and 5-sec exploration time conditions were collapsed and 11 subjects were randomly selected from these two conditions. Two of the three ANOVAS conducted on these data revealed significant effects of type of movement. The effect of the unlimited time condition was significant $[F(2,20)=32.56$, $\left.M S_{\mathrm{e}}=98.947, p<.001\right]$. Post hoc contrasts revealed a significant difference between enclosure (global and part) and contour following. The ANOVA performed on the 3- and 5-sec conditions revealed a significant main effect of movement $\left[F(2,20)=117.86, M S_{\mathrm{e}}=36.2979\right.$, $p<.001]$ and an interaction between exploration time and movement $\left[F(2,20)=59.591, M S_{\mathrm{e}}=8.78, p<\right.$ $.001]$. The third ANOVA, on the 1.5-sec condition, revealed no significant effects. As Figure 6 shows, limiting the exploration time reduced the time spent to explore object contours. This time goes from $4.9 \mathrm{sec}$ under the unlimited time condition to $2.5 \mathrm{sec}$ in the $3-5-\mathrm{sec}$ condition, and to 0.6 seconds in the $1.5-\mathrm{sec}$ condition.

As can be seen in Figure 6, the time necessary to detect whether the object was symmetric or asymmetric did not differ at any of the exploration time conditions. This finding agrees with our previous results with raised shapes, but differs from results reported by Locher and Simmons (1978), who used large raised polygons. However, the size of the polygons used in the Locher and Simmons studies was very large, requiring a sequential mode of exploration.

Frequency distributions by position in the total hand movements sequence as a function of exploration time. Figure 7 displays the cumulative percentage of occurrence of the three main hand movements observed as a function of its order number in the total sequence of movements and exploration times. Note that the number of positions in the sequence diminished with the reduction of exploration time (up to six positions in the unlimited time condition to just three in the $1.5-\mathrm{sec}$ 


\section{UNLIMITED TIME}

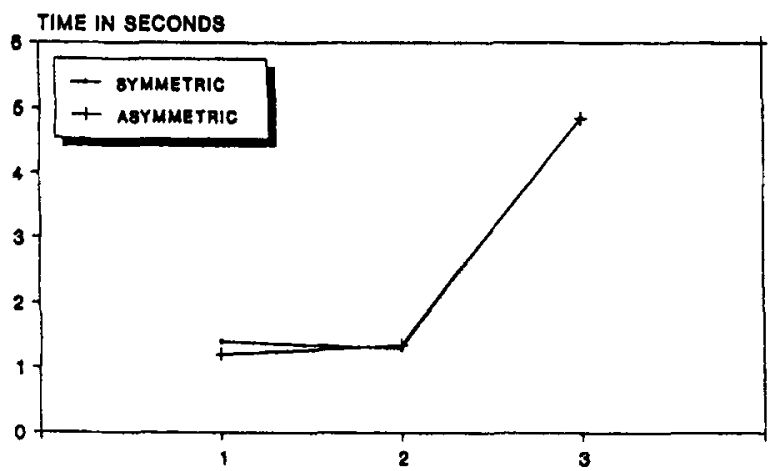

\section{3-5 \& EXPLORATION TIME}

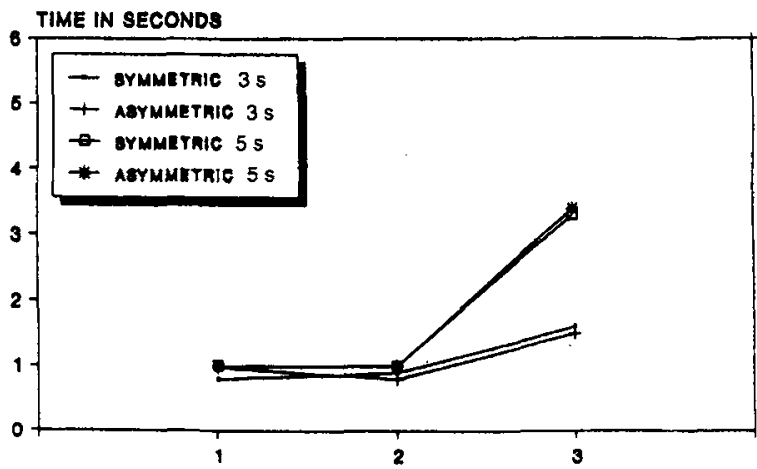

\section{5 s EXPLORATION TIME}

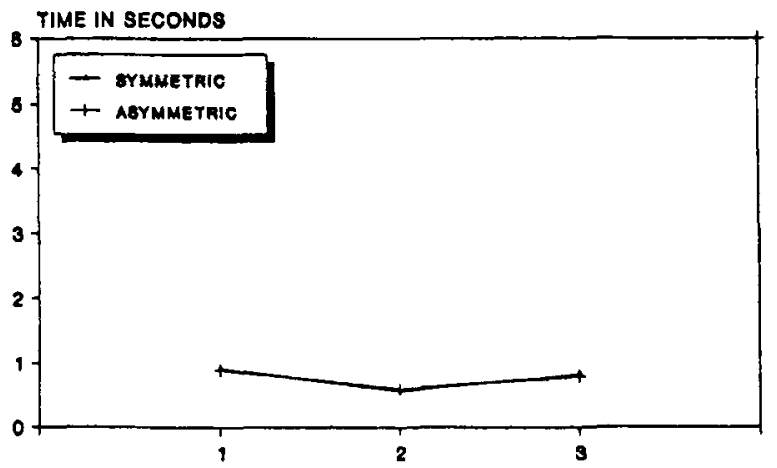

Figure 6. Mean times dedicated to the different exploratory procedures under unlimited time, 5,3 , and 1.5 sec.

condition). This figure reflects the order in which the different movements were executed. For example, global enclosure was mostly executed in the first position of the sequence. Contour following appeared more often in Position 2, interconnected with enclosure (part). However, as exploration time decreased (1.5-sec condition), contour following appeared as the second movement of the sequence $98 \%$ of the time. The proportion of global enclosure produced in the first position also increased considerably, showing it to be very efficient as well as fast. As in Lederman and Klatzky's (1990) studies on haptic classification of common objects, this was the steepest curve across exploration times. It usually occurred in the first position of the sequence $(n=877$ across exploration times).

The other two types of hand movements observed, enclosure (part) and contour following, tended to occur in alternation in the second and third positions of the sequence after global enclosure. However, enclosure (part) was much less frequent than contour following ( $n=454$ and $n=932$, respectively). As noted, only a few objects had salient parts. In the 1.5 -sec condition, contour following occurred $98 \%$ of the time in the second position, after global enclosure, and sometimes after enclosure (part). This movement was associated only with objects that exhibited noticeable and salient parts.
This pattern of results clearly supports Lederman and Klatzky's $(1987,1990)$ proposal that the type of movements performed with 3-D objects are dictated by the object properties that the system must process. By showing that the hand movements observed in the haptic symmetry detection task were almost exclusively the three exploratory procedures associated with processing structural properties of shape, we have extended Lederman and Klatzky's results with multidimensional common objects to the domain of haptic perception of the bilateral symmetry of unfamiliar objects.

Hand movements: Most frequent sequences. The proportions of the most frequent sequences of hand movements that occurred as a function of exploration time and number of movements in the sequence are displayed in Table 5. Three main features are notable: (1) Exploration began with an enclosure followed by another movement; (2) the most common pattern (near half of the total number of sequences) was one composed of just two exploratory movements, global enclosure followed by contour following; (3) as exploration time decreased $(1.5 \mathrm{sec})$, the number of movements in the sequence also decreased, and the number of trials in which just one movement was performed increased considerably (31\%); in this case, the movement was global enclosure (26\%) and less often, enclosure (part) $(5 \%)$. 


\section{UNLIMITED TIME}

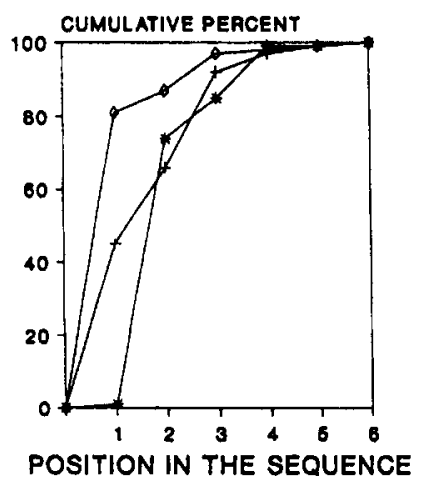

\section{S EXPLORATION TIME}

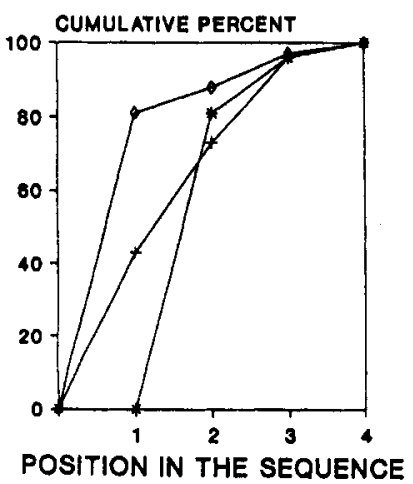

5 s EXPLORATION TIME

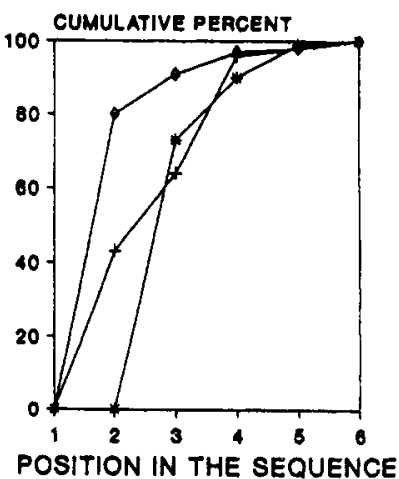

TYPE OF MOVEMENTS

$\rightarrow$ QLOBAL ENCLOSURE

+ Partial enclosure

* CONTOUA FOLLOWING

\section{5 \& EXPLORATION TIME}

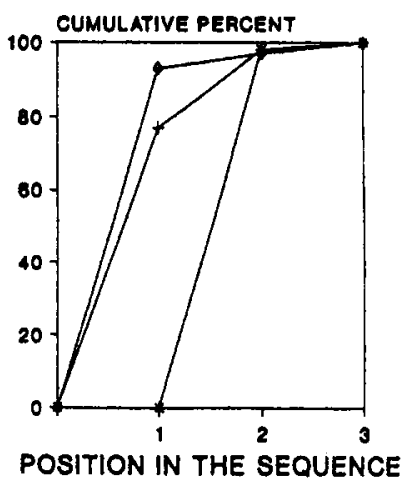

Figure 7. Cumulative percentage of occurrence of hand movements as a function of position in the total sequence of movements and exploration times.

A second scorer was trained by the first scorer and by one of the authors. She scored the frequency of all types of hand movement for one fourth of the trials. The time scores for each movement by the two scorers was highly correlated $(91 \%)$.

\section{Experiment 4}

The aim of Experiment 4 was to test further the reference frame hypothesis with objects. Bimanual exploration was compared with unimanual (preferred hand) exploration. We reasoned that disrupting the spatial frame created by reference to the body midaxis and not allowing the execution of two parallel movements would hinder haptic detection of bilateral symmetry. The hypothesis was that the advantage in performance with symmetric objects would be reduced in unimanual exploration. In this experiment, observers explored the objects with the preferred hand. Accuracy scores obtained from this group were compared with accuracy scores from the free exploration group in Experiment 3.

\section{Method}

Subjects. Twenty new observers from the same pool as those in previous experiments participated in this study.

Stimuli. The stimuli were the same objects as those in Experiment 3.

Procedure. The only change in the procedure was to instruct the subject that the object would be explored using his/her preferred hand only. Exploration time was not constrained. The rest of the procedure was the same as that in Experiment 3.

\section{Results and Discussion}

Accuracy analysis. The detection of symmetry by the haptic system using just a hand was quite good. The mean percentage correct was $88 \%(S D=11)$. The mean proportions of correct detection for symmetric and asymmetric objects were $90 \%$ and $86 \%$, respectively.

The ANOVA on accuracy for scores for the 2 (modes of exploration: bimanual and unimanual) $\times 2$ (type of objects: symmetric and asymmetric) factorial with repeated measures in the last factor showed a significant main effect of symmetry $\left[F(1,38)=8.07, M S_{\mathrm{e}}=85.44, p<.001\right]$. This meant that haptic detection of symmetric $3-\mathrm{D}$ objects 
Table 5

Most Frequent Sequences of Hand Movements

\begin{tabular}{lccc}
\hline & \multicolumn{2}{c}{ Exploration Time Condition } \\
\cline { 2 - 4 } Sequence & Unlimited & $3-5 \mathrm{sec}$ & $1.5 \mathrm{sec}$ \\
\hline & One movement & - & .26 \\
Enc (G) & .04 & - & .05 \\
Enc (P) & - & & \\
& Two movements & .52 \\
Enc (G), CF & .41 & .50 & .07 \\
Enc (P), CF & .14 & .09 & - \\
& & & - \\
Enc (G), Enc (P), CF & .08 & .08 & - \\
Enc (G), CF, Enc (P) & .04 & .09 & - \\
Enc (G), CF, Enc (G) & .04 & .09 & .09 \\
Enc (P), Enc (G), CF & .04 & & .10 \\
Other sequences all & & .10 & \\
starting with Enc & .21 & & \\
\hline
\end{tabular}

Note-Enc (G), enclosure (global); Enc (P), enclosure (part); CF, contour following. Data from 3- and 5-sec exploration time conditions were collapsed.

was more accurate than that of asymmetric objects even when exploration was unimanual. Mode of exploration was not statistically significant $(F<1)$; symmetry did not interact with mode of exploration $(F<1)$.

Sensitivity and bias analysis. The $d_{\text {avg }}^{\prime}$ for the unimanual group was 3.02 . The bias criterion $c$ was -.22 and showed no response bias. The sensitivity index between bimanual and unimanual exploration did not differ significantly.

Contrary to our prediction, the haptic detection of object symmetry was not disrupted under unimanual exploration. However, this result does not rule out the reference frame hypothesis as an explanatory factor.

\section{Experiment 5}

On the basis of the results from the hand movement analysis, Experiment 5 was designed to test the hypothesis that performing just a global enclosure movement would be sufficient to detect bilateral symmetry of 3-D objects. The only movement allowed was an enclosing movement consisting of molding the hands around the object. Hand movements were videotaped to ascertain that only this type of movement was performed in each trial.

\section{Method}

Subjects. Twenty new right-handed subjects from the same pool volunteered. In addition, the data from the 20 subjects in the unrestricted exploration time condition who participated in Experiment 3 were used in the statistical analysis.

Stimuli. The stimuli were the same wooden objects as those used in Experiments 3 and 4.

Procedure. The procedure was the same as that in Experiments 3 and 4 except that the type of exploration movement allowed was just an enclosure movement per trial.

\section{Results and Discussion}

Accuracy analysis. Overall accuracy when movement was restricted to just an enclosure was quite good (85\%).
Mean accuracy scores for symmetric and asymmetric objects were $89 \%$ and $80 \%$, respectively.

An ANOVA on accuracy corresponding to the 2 (type of movements: unrestricted and enclosure) $\times 2$ (type of objects: symmetric and asymmetric) factorial with repeated measures in the last factor showed a significant main effect of movement $\left[F(1,38)=4.317, M S_{\mathrm{e}}=\right.$ $104.54, p<.05]$. The mean accuracy for the unrestricted movements group was $90 \%$, versus $85 \%$ for the enclosure group. As in previous experiments with 3-D objects, symmetric judgments were more accurate than were asymmetric ones $\left[F(1,38)=10.63, M S_{\mathrm{e}}=120.39, p<.001\right]$. The interaction of movements $\times$ type of objects was not significant $(F<1)$.

Sensitivity and bias analysis. The $d_{\text {avg }}^{\prime}$ for the enclosure group was 2.58 , statistically above chance. The bias index $c$ was -.16 and did not show bias. In line with the accuracy ANOVA analysis, the sensitivity of the two groups was significantly different-higher in the free exploration group than in the enclosure group $(p<.05)$.

The results showed that two-handed haptic perception of symmetry for unfamiliar objects that can be enclosed in the hand(s) is excellent. However, even though performance was outstanding when movement was restricted to just an enclosure, free exploration produced even better performance. This suggests that the most common second movement performed during haptic exploration (contour following) is effective in the detection of this property of shape.

\section{GENERAL DISCUSSION}

The current study addressed several issues concerning haptic shape and object perception. The main conclusion is that the haptic system is very accurate, especially when the stimuli are 3-D objects. Through active touch, subjects extract important spatial information of the explored stimuli, such as its symmetry or asymmetry. This information is extracted essentially during a very early step of haptic exploration. Furthermore, bimanual exploration is superior to unimanual exploration, probably due to parallel processing and the possibility of using body-centered reference frames. In addition, the types as well as the sequences of exploratory movements allow one to know what the relevant information is for haptic judgments. Since most haptic studies have used raised shapes, the use of 3-D objects to test properties of the haptic processing system may be promising.

The present experiments explicitly investigated the accuracy and sensitivity of the haptic system in detecting bilateral symmetry. We also studied the effects of restricting haptic exploration time and different types of scanning (one vs. two fingers and global enclosure) in relation to the perceiver's body reference axis.

The results of Experiments 1 and 2 showed repeatedly that bilateral symmetry/asymmetry of raised-line shapes can be detected haptically. A quite clear-cut pattern of results has emerged. Four main results from these experiments should be emphasized: First, haptic percep- 
tion was at least moderately sensitive in detecting symmetry in unfamiliar, simple raised-line shapes. Second, observers were consistently far more accurate in detecting asymmetric shapes than symmetric ones. Third, performance was almost equal across differences in exploration time. Interestingly, however, the asymmetric judgments were consistently more accurate than symmetric judgments even when exploration time was restricted to $2 \mathrm{sec}$. Fourth, bimanual exploration was consistently better than unimanual exploration and did not differ between the left and the right hand. It has been assumed that the right hemisphere is superior for spatial processing (see, e.g., Bradshaw \& Nettleton, 1983; Stein, 1992). There are reasons to expect left-hand advantage for spatial tasks, but hand superiority in tactual perception also tends to vary with tasks (Bertelson, 1982; Millar, 1984). Our results did not show an advantage for the left hand in accuracy, and although we previously found faster left-hand processing, the left hand was not more accurate than the right hand (Ballesteros, Manga, Navarredonda, \& Reales, 1993).

The signal detection analysis revealed that sensitivity measured by $d^{\prime}$ showed at least a moderate capacity of touch to perform the symmetry/asymmetry judgment task with raised displays. However, our results on criterion did not allow us to conclude that there was no response bias, although the response bias was not statistically significant under unlimited exploration time (Experiment 1) or in the two-finger exploration condition (Experiment 2). When exploration time was restricted, sensitivity did not change, but response bias to say "asymmetric" appeared. SDT analysis performed on data from Experiment 2 showed that the degree of sensitivity did not differ under unimanual and bimanual modes of exploration. Sensitivity in two-finger exploration (one from each hand) was not different from that in single-finger exploration, but the response bias found in the unimanual condition disappeared under bimanual exploration. Thus, it is unlikely that response bias could explain the findings, because the more intriguing findings on haptic processing of symmetry were not invalidated. As noted, there was no significant response bias in the main conditions, which showed better judgments of asymmetric stimuli.

Our results demonstrate that for tasks that demand judgments of symmetry, there was consistently better detection for asymmetric than for bilaterally symmetric shapes when one finger was used over a range of exploration times. Two-finger exploration reduced but did not eliminate this asymmetry. This mode of exploration produced an increase in the detection of symmetry but did not change asymmetry.

This finding is consistent with results from a series of experiments conducted in collaboration with Susanna Millar (Ballesteros et al., 1996; Millar, Ballesteros, \& Reales, 1994). We tested for implicit effects of symmetry in that study. Half the symmetric and asymmetric shapes (similar to those used here) contained a closed shape that had to be detected. Scanning by the two forefingers, previously aligned to the body midline, showed greater accuracy for (vertical) symmetric open shapes. Scanning by one finger showed no effect of symmetry, consistent with a previous implicit test of symmetry (Millar, 1978). With the explicit detection of symmetry that was demanded by the present task, we showed that the dimension is also detected with scanning by one finger, but that asymmetric features are easier. The findings are consistent with the hypothesis that the advantage of symmetric shapes depends on spatial reference information. Millar (1981, 1985) distinguished experimentally between movement coding and coding by self-referent spatial axes in smallscale space. Visuospatial experience tended to favor reliance on external spatial frames. In the absence of visual experience or with reduced external cues, body-centered spatial reference frames need to be reliable to provide reference for shape reading. When there are few spatial anchors, as in blind exploration by one finger, symmetry is not perceptually salient because scanning is sequential and feature cues need to be integrated for global shape perception. When detection of symmetry is demanded in these conditions, asymmetric shapes are easier because integration does not have to be accomplished first. Additional spatial reference (e.g., fingers to midline) provide anchoring organization that favors symmetric shapes. In these experiments, subjects judged whether a shape (similar to those used in the present experiments) was open or closed. Half of the shapes in each category were symmetrical and half were asymmetrical.

From exploring raised shapes to exploring 3-D objects. Because the 2-D and 3-D stimuli were not comparable, any comparisons must be considered tentative. The results suggest different patterns of performance. The 3-D objects allow subjects to use global simultaneous processing by using the hands to enclose the object. Performance with 3-D objects was very accurate even under severe time constraints $(1.5 \mathrm{sec})$.

But how does the haptic perceptual system perform the detection task? Videotaped data provided some answers to this question. To study the haptic processing of symmetry, a task was devised to be performed with the above-mentioned two types of stimuli.

As Klatzky and Lederman have shown in several studies, the motor component enhances the perceptual capability of the "intelligent" hand. What happens with objects is that the hands manipulate them in a global manner. The first contact is enclosure, defined as molding hands and fingers to the maximum possible of object's contour (Klatzky \& Lederman, 1987). With this hand movement, the perceiver tries to capture global information from all the parts from the beginning of the exploration.

A second, also very common, way of object exploration is contour following, a dynamic way of exploring along the edges of the object. As Lederman and Klatzky $(1987,1990)$ noted, these two exploratory procedures are critically important to haptic perception of shape. It 
is likely, they suggested, that the motor subsystem of the hand used to manipulate the objects enhances the sensory subsystem composed of cutaneous, thermal, and kinesthesic sensors. In this sense, raised-line stimuli reduce the effectiveness of the system, forcing it to use only a very small part of its encoding capability, denying it use of the system's motor component, and thereby limiting its performance. We argued that our wooden objects enhanced the motor subsystem of the hand, allowing the perceiver to make contact with many parts of the object simultaneously (Ballesteros, 1993). This is due to the increase of the sources of kinesthesic information-the larger number of kinesthesic receptors activated in 3-D object exploration.

Experiments more directly focused on comparing the two sets of shapes through the use of 2-D bilaterally symmetrical shapes extended uniformly in the third dimension will be necessary before any conclusions and possible generalizations can be drawn.

\section{REFERENCES}

APPELLE, S. (1991). Haptic perception of form: Activity and stimulus attributes. In M. A. Heller \& W. Schiff (Eds.), The psychology of touch (pp. 169-188). Hillsdale, NJ: Erlbaum.

BALlesteros, S. (1993, May). Haptic perception and types of memory for raised patterns and 3-dimensional objects. Paper presented at the International Conference on Object Representation in Visual and Haptic Systems, Madrid.

BAllesteros, S., \& CoOPER, L. A. (1992, July). Perceptual priming of two-dimensional patterns following visual presentation. Paper presented at the 33rd International Congress of Psychology, Brussels.

Ballesteros, S., ManGa, D., Navarredonda, A. B., \& Reales, J. M. (1993, November). Perceptual asymmetry in haptic discrimination. Paper presented at the 34th annual meeting of the Psychonomic Society, Washington, DC.

Ballesteros, S., Millar, S., \& Reales, J. M. (1996). Symmetry in haptic and visual shape perception. Manuscript submitted for publication.

BERTELSON, P. (1982). Lateral differences in normal man and lateralization of brain functions. International Journal of Psychology, 17, 173-210.

Bolanowski, Jr., S. J., Gescheider, G. A., Verrillo, R. T., \& CheCKosKY, C. M. (1988). Four channels mediate the mechanical aspects of touch. Journal of the Acoustical Society of America, 84, 1680-1694.

Bradshaw, J. L., \& Nettleton, N. C. (1983). Human cerebral asymmetry. Englewood Cliffs, NJ: Prentice-Hall.

CoOper, L. A., Schacter, D. L., Ballesteros, S., \& Moore, C. (1992). Priming and recognition of transformed three-dimensional objects: Effects of size and reflection. Journal of Experimental Psychology: Learning, Memory, \& Cognition, 18, 43-57.

Craig, J. C. (1985). Attending to two fingers: Two hands are better than one. Perception \& Psychophysics, 38, 496-511.

Davidson, P. W. (1972). Haptic judgment of curvature by blind and sighted humans. Journal of Experimental Psychology, 93, 43-55.

Davidson, P. W., Aвbott, S., \& Gershenfeld, J. (1974). Influence of exploration time on haptic and visual matching of complex shape. Perception \& Psychophysics, 15, 539-543.

Garbin, C. P., \& Bernstein, I. H. (1984). Visual and haptic perception of 3-dimensional solid forms. Perception \& Psychophysics, 36, 104-110.

Gibson, J. J. (1962). Observation on active touch. Psychological Review, 69, 477-490.

HELLER, M. A. (1992). The effect of orientation on tactual braille recognition: Optimal touching positions. Perception \& Psychophysics, 51, 549-556.

KapPers, A. M. L., Koenderink, J. J., \& LichtenegGer, I. (1994).
Haptic identification of curved surfaces. Perception \& Psychophysics, 56, 53-61.

Kappers, A. M. L., Koenderink, J. J., \& te Pas, S. F. (1994). Haptic discrimination of doubly curved surfaces. Perception, 23, 1483-1490.

KATZ, D. (1989). The world of touch (L. E. Krueger, Trans.). Hillsdale, NJ: Erlbaum. (Original work published 1925)

Klatzky, R. L., \& Lederman, S. J. (1987). The intelligent hand. In G. H. Bower (Ed.), The psychology of learning and motivation (Vol.21, pp. 121-151). San Diego: Academic Press.

KlATzKY, R. L., \& Lederman, S. J. (1992). Stages of manual exploration in haptic object identification. Perception \& Psychophysics, 52, 661-670.

KLATZKy, R. L., LEDERMAN, S. J., \& METZGER, V. A. (1985). Identifying objects by touch: An "expert system." Perception \& Psychophysics, 37, 299-302.

KLATZKY, R. L., LederMaN, S. J., \& ReED, C. (1987). There's more to touch than meets the eye: The salience of object attributes for haptics with and without vision. Journal of Experimental Psychology: General, 116, 356-369.

KiatzKy, R. L., LoOmis, J. M., Lederman, S. J., WaKe, H., \& Fujita, N. (1993). Haptic identification of objects and their depictions. Perception \& Psychophysics, 54, 170-178.

LAPPIN, J. S., \& FoULKE, E. (1973). Expanding the tactual field of view. Perception \& Psychophysics, 14, 237-241.

LEDERMan, S. J., \& KLATZKY, R. L. (1987). Hand movements: A window into haptic object recognition. Cognitive Psychology, 19, 342-368.

LEDERMAN, S. J., \& KLATZKY, R. L. (1990). Haptic classification of common objects: Knowledge-driven exploration. Cognitive Psychology, 22, 421-459.

Locher, P. J., \& Simmons, R. W. (1978). Influence of stimulus symmetry and complexity upon haptic scanning strategies during detection, learning, and recognition tasks. Perception \& Psychophysics, 23, 110-116.

Loomis, J. M. (1990). A model of character recognition and legibility. Journal of Experimental Psychology: Human Perception \& Performance, 16, 101-120.

Loomis, J. M., \& Lederman, S. J. (1986). Tactual perception. In K. R. Boff, L. Kaufman, \& J. P. Thomas (Eds.), Handbook of perception and human performance (Vol. 2, pp. 31-1 to 31-44). New York: Wiley.

MacMillan, N. A., \& Creelman, C. D. (1991). Detection theory: A user's guide. Cambridge: Cambridge University Press.

Millar, S. (1978). Aspects of memory for information from touch and movement. In G. E. Gordon (Ed.), Active touch (pp. 215-227). Oxford, U.K.: Pergamon.

Millar, S. (1979). The utilization of external and movement cues in simple spatial tasks by blind and sighted children. Perception, $\mathbf{8}$, 11-20.

Millar, S. (1981). Self-referent and movement cues in coding spatial locations by blind and sighted children. Perception, 10, 255-264

MILLAR, S. (1984). Is there a "best hand" for braille? Cortex, 20, 75-87.

Millar, S. (1985). Movement cues and body orientation in recall of locations by blind and sighted children. Quarterly Journal of Experimental Psychology, 37A, 257-279.

MillaR, S. (1994). Understanding and representing space. Oxford: Oxford University Press.

Millar, S., Ballesteros, S., \& Reales, J. M. (1994, November). Influence of symmetry in visual and haptic perception. Paper presented at the 35th annual meeting of the Psychonomic Society, St. Louis.

OLDFIELD, R. C. (1971). The assessment and analysis of handedness: The Edinburgh inventory. Neuropsychologia, 9, 97-113.

REALES, J. M., \& BALLESTEROS, S. (1994). SDT_SP, a program in Pascal for computing parameters and significance tests from several detection theory designs. Behavior Research Methods, Instruments, \& Computers, 26, 151-155.

REALES, J. M., \& BALleSTEROS, S. (1995). TDS, un programa de ordenador para la teoria de detección de señales. Madrid: Editorial Universitas.

RÉvÉsz, G. (1950). Psychology and art of the blind. London: Longmans Green.

Schacter, D. L., CoOPer, L. A., \& Delaney, S. M. (1990). Implicit 
memory for unfamiliar objects depends on access to structural descriptions. Journal of Experimental Psychology: General, 119, 5-24.

Simmons, R. W., \& LOCHER, P. J. (1979). The role of extended perceptual experience upon haptic perception of nonrepresentational shapes. Perceptual \& Motor Skills, 48, 987-991.

STEIN, J. F. (1992). The representation of egocentric space in the posterior parietal cortex. Behavioral \& Brain Sciences, 15, 691-700.

ZINCHENKO, V. P., \& LoMOV, B. F. (1960). The functions of hand and eye movements in the process of perception. Voprosy Psikhologi, 1 , 12-26.

\section{NOTE}

1. We ran another condition in which the same tactual shapes were presented as raised dots made in cardboard in an unrestricted exploration time condition. Each dot's diameter was $1.1 \mathrm{~mm}$ wide and $.65 \mathrm{~mm}$ high. The separation between dots was $1 \mathrm{~mm}$. The results did not differ from those obtained with the raised lines.

(Manuscript received September 23, 1994; revision accepted for publication February 22, 1996.) 